\title{
Adenocarcinoma Arising From a Gastric Duplication Cyst With Lymph Node Metastasis
}

\author{
Shoichi Kinugasa ${ }^{1}$, Hiroyuki Monma ${ }^{1}$, Yoshio Sakamoto ${ }^{2}$, Takafumi Watanabe ${ }^{2}$, Masayo Fujimoto ${ }^{3}$
}

1. Surgery, Hyogo Prefectural Kakogawa Medical Center, Kakogawa, JPN 2. Gastroenterology, Hyogo Prefectural Kakogawa Medical Center, Kakogawa, JPN 3. Pathology, Hyogo Prefectural Kakogawa Medical Center, Kakogawa, JPN

Corresponding author: Shoichi Kinugasa, skinugasa@yahoo.co.jp

\begin{abstract}
Gastric duplication cysts (GDCs) are a relatively rare congenital anomalies and are mostly diagnosed in the early years of life. Herein, we report a very rare surgical case of adenocarcinoma arising from a GDC with lymph node metastasis. A 78-year-old woman was referred to our hospital because of elevated serum levels of cancer antigen (CA) 19-9. Endoscopic ultrasound, contrast fistulography, and computed tomography showed a cystic lesion communicating with the lesser curvature of the stomach. The serum levels of CA 19-9 were high, and fluorine-18 fluorodeoxyglucose positron emission tomography/computed tomography (18FFDG PET/CT) imaging demonstrated a slightly enlarged lymph node with high FDG uptake after four months. The size of the cyst was unchanged. It was diagnosed as a GDC. The enlarged lymph node was highly likely to be malignant. Hence, we performed a distal gastrectomy involving the origin of entry and whole body of the GDC with en bloc regional lymphadenectomy. The postoperative pathology was consistent with GDC with moderately differentiated adenocarcinoma and lymph node metastasis. Adjuvant chemotherapy with tegafur-gimeracil-oteracil potassium (S-1) was administered for 12 months. At present, the patient is alive, with no recurrence of the lesion even four years after the operation. GDCs in adults are rare and may predispose to malignancy. Early diagnosis and prompt surgical intervention are important for favorable outcomes.
\end{abstract}

Categories: Gastroenterology, General Surgery, Oncology

Keywords: gastric duplication cyst, adenocarcinoma, malignancy, gastrectomy, surgery

\section{Introduction}

Gastric duplication cysts (GDCs) are relatively rare congenital anomalies that are mostly diagnosed in the early years of life. It is usually diagnosed before age 12. GDCs in adults are generally detected incidentally. This incidental detection is also because most cases are asymptomatic though in some cases, a symptomatic obstruction may occur. One-third of GDCs are associated with other developmental anomalies such as GDCs at other locations and duodenal diverticula [1]. Adenocarcinoma rarely arises from a GDC, and only 16 cases of malignancy have been reported to date [2-16]. An established resection procedure for and the range of this malignancy have not been discovered thus far. We present a case of a patient showing long-term survival after gastrectomy and lymph node dissection for adenocarcinoma arising from a GDC with a metastatic lymph node at the lesser curvature of the stomach.

Review began 12/17/2020 Review ended 12/22/2020 Published 12/27/2020

\section{() Copyright 2020}

Kinugasa et al. This is an open access article distributed under the terms of the Creative Commons Attribution License CC-BY 4.0., which permits unrestricted use, distribution, and reproduction in any medium, provided the original author and source are credited.

\section{Case Presentation}

A 78-year-old woman was referred to our hospital because of incidentally elevated serum levels of cancer antigen (CA) 19-9 by medical check including cancer screening with no significant clinical symptoms. She had a history of hypertension, but she had no other significant past history nor family history of malignancy. She was diagnosed with a Helicobacter pylori infection by a stool antigen test and subsequently underwent eradication therapy just before her visit to our hospital and it was successful. All laboratory test results were normal except the CA $19-9$ concentration, which was $186.9 \mathrm{U} / \mathrm{mL}$ (normal, <37 U/mL). The patient underwent serial diagnostic imaging examinations of the alimentary system. Upper gastrointestinal endoscopy revealed a small orifice in the lesser curvature of the lower body of the stomach (Figure $1 \mathrm{~A}$ ). Suction cytology from the hole did not detect any malignant cells. Endoscopic ultrasound (EUS) and contrast fistulography through the hole showed a tubular lesion lined by a mucosal layer contiguous with that of the stomach, and the surrounding muscularis propria was visible in the root of the GDC (Figure $1 B, 1 C$ ). In the distal portion of the cyst, the wall of the GDC lacked the mucosal layer and muscularis propria. Abdominal computed tomography (CT) showed a cystic lesion along the lesser curvature of the stomach and lymph node swelling. We followed up these lesions using CT because the patient did not report any discomfort. The serum levels of CA 19-9 increased to $512.4 \mathrm{U} / \mathrm{mL}$ four months later. Fluorine-18 fluorodeoxyglucose positron emission tomography/CT (18F-FDG PET/CT) imaging demonstrated a slightly enlarged lymph node with high FDG uptake (maximum standardized uptake value [SUVmax] = 5.6). The size of the cyst was unchanged (SUVmax = 3.9) (Figure 2). It was determined that the GDC and enlarged lymph node were highly likely to be malignant. Resection of the primary lesion with negative surgical margins and lymphadenectomy were considered necessary. Laparotomy revealed a cystic mass that originated from the angle of the lesser curvature of the stomach and was growing toward the liver. A swollen hard lymph node, 


\section{Cureus}

regional right cardiac node, was located at the tip of the cystic lesion adjacent to the liver. The peritoneal lavage fluid that was collected and examined immediately after laparotomy was found to be cytology negative. We performed a distal gastrectomy involving the orifice and entire body of the GDC $(11 \mathrm{~cm}$ in length), with en bloc D2 regional lymphadenectomy and Billroth 1 reconstruction, and the patient was discharged two weeks after the surgery (Figure 3). The postoperative pathology was consistent with GDC with moderately differentiated adenocarcinoma and a lymph node metastasis out of 34 dissected nodes with lymphatic and blood vessel invasion. The cancer cells were confined to the mucosa of the root of the GDC. In the distal part of the GDC, the cancer cells were present in the damaged layered structure of the GDC, and the depth of the tumor was determined as T3 (Figure 4). The final tumor-node-metastasis stage was pT3N1M0, stage IIB. Adjuvant chemotherapy with tegafur-gimeracil-oteracil potassium (S-1) was administered for 12 months. The patient has been followed up at our hospital every six months with CT in combination with measurement of CA-19 and annual endoscopy. At present, the patient is alive without any recurrence of the lesion even four years after the operation.

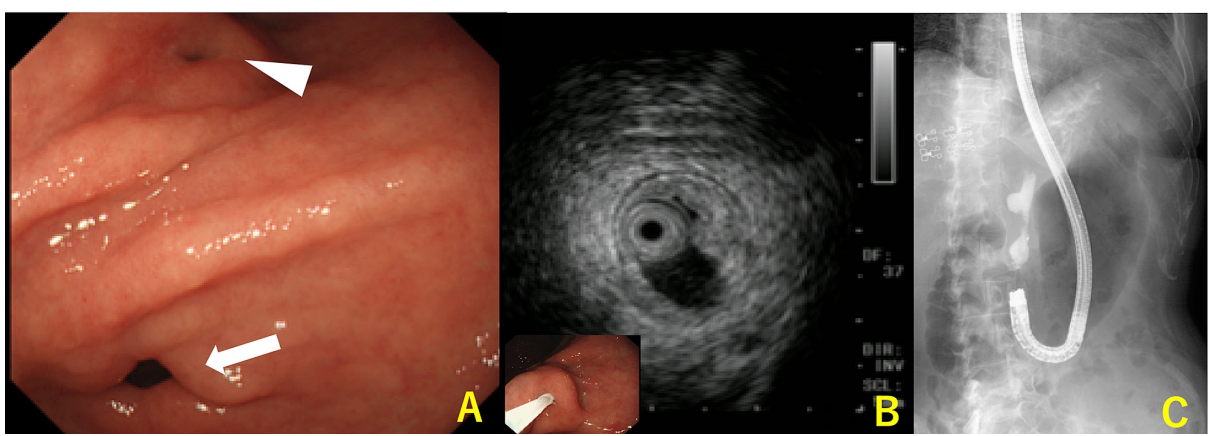

\section{FIGURE 1: Pre-operative images}

Upper gastrointestinal endoscopy shows a small orifice along the lesser curvature of the lower body of the stomach; the pyloric ring (arrow) and orifice of the duplication cyst (arrowhead) are seen (A). Endoscopic ultrasound shows a tubular lesion lined by a mucosal layer with the surrounding muscularis propria visible in the root of the gastric cyst, suggestive of a duplication cyst (B). Contrast fistulography shows a tubular lesion, $7 \mathrm{~cm}$ in length, with a stricture in the middle portion of the lesion (C).

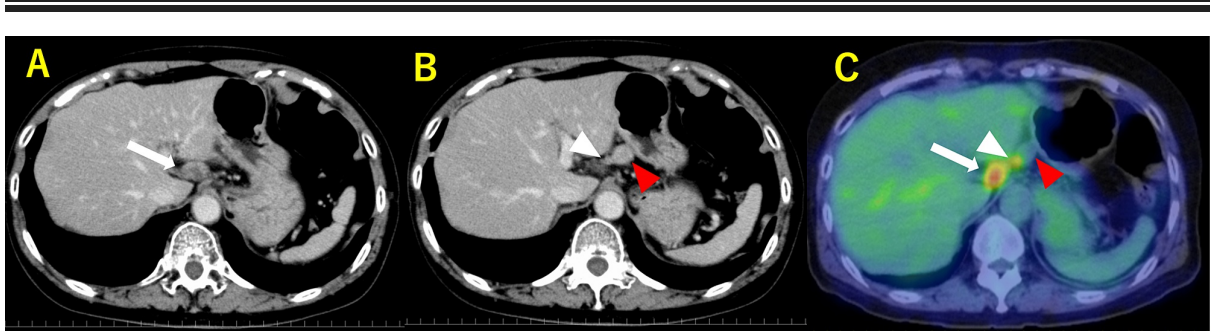

\section{FIGURE 2: CT and PET/CT}

Pre-operative enhanced computed tomography (A,B) and Fluorine-18 fluorodeoxyglucose (FDG) positron emission tomography/computed tomography imaging (C) demonstrates a slightly enlarged lymph node with high FDG uptake (SUVmax $=5.6$, white arrow). The size of the cyst (red arrowhead) was unchanged. FDG uptake (SUVmax $=3.9$, white arrowhead) showed in stricture region.

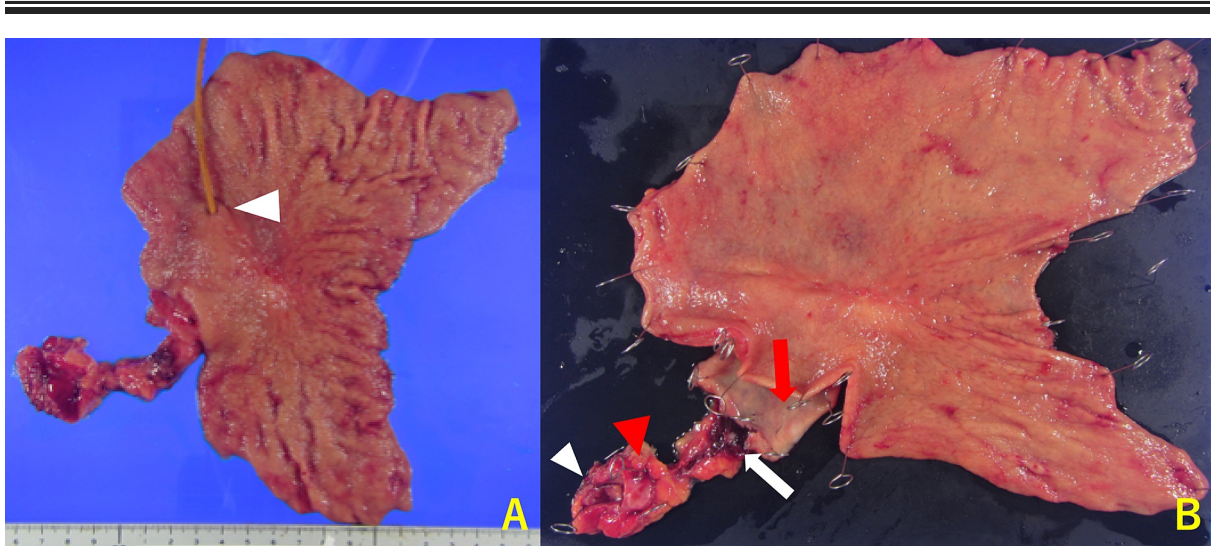

FIGURE 3: Resected specimen 


\section{Cureus}

Resected specimen: the orifice of the duplication cyst (white arrowhead) (A); macroscopic view: the mucosa surface of the duplication cyst (red arrow), stricture revealing the cancer cells (white arrow), blind end of the duplication cyst (red arrowhead), metastatic lymph node (white arrowhead) (B).

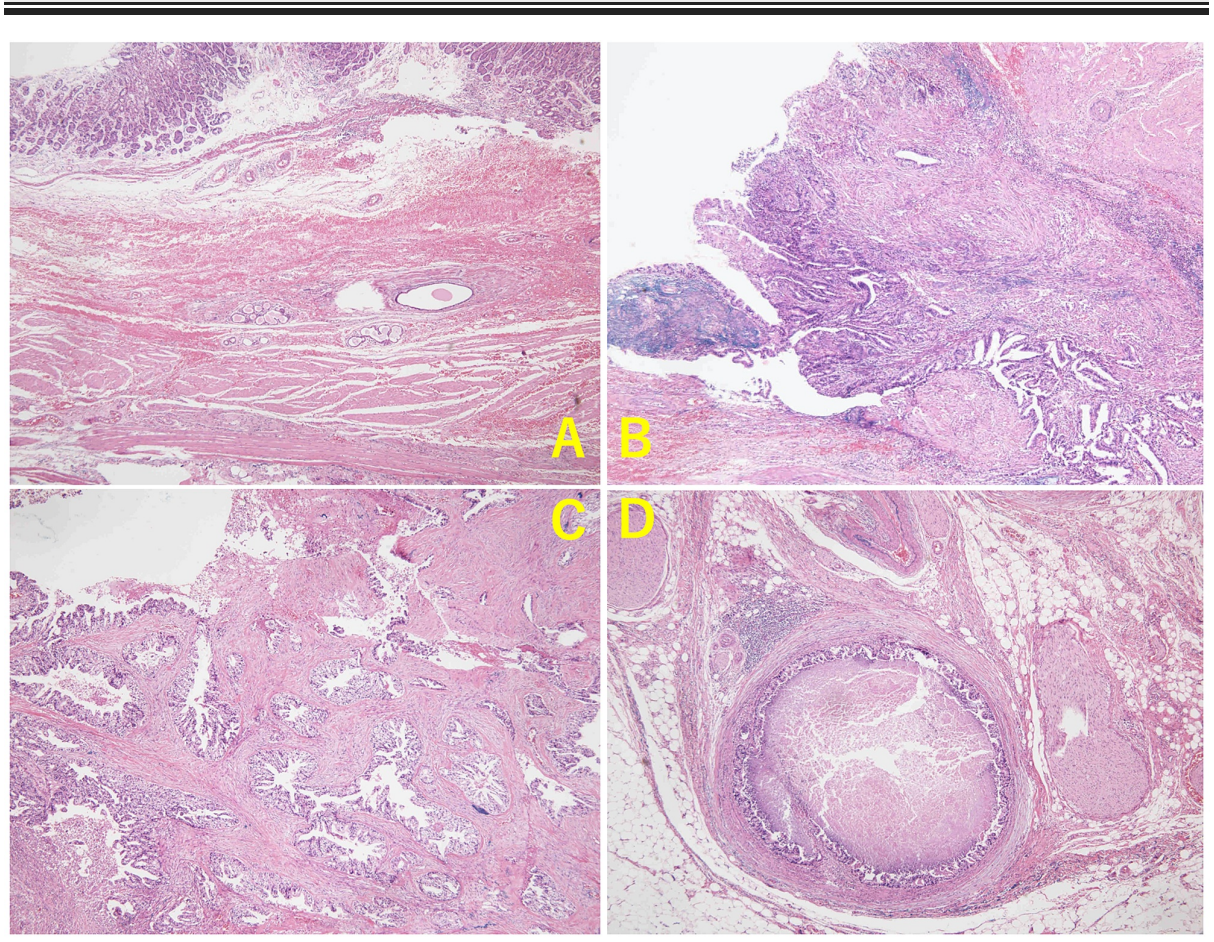

\section{FIGURE 4: Microscopic findings of a gastric duplication cyst}

The gastric side of the cystic wall has an inner mucosal lining, the submucosa, and muscularis propria contiguous with that of the gastric wall (A). Moderately differentiated adenocarcinoma confined to the mucosal layer is mainly observed in the proximal part of the duplication cyst (B); deep infiltration by the cancer cells; the layered structure of the cystic wall disappears in the distal part of the duplication cyst (C); vascular invasion (D).

\section{Discussion}

This report reveals that GDCs in adults are rare and may predispose to malignancy. Early diagnosis and prompt surgical intervention are important for favorable outcomes. The term 'alimentary tract duplication' was used by Ladd to describe congenital malformations that involve the mesenteric side of the associated alimentary tract and share a common blood supply with the native bowel [17]. Alimentary tract duplications are rare and occur anywhere along the digestive tract in one in 4500 births [18]. The most common locations of duplication are the small intestine, esophagus, and colon. GDCs represent $4 \%$ of all duplication cysts [15]. The typical features of GDCs are as follows: a) the cyst wall is contiguous with the stomach wall; (b) the cyst is surrounded by smooth muscle, which is contiguous with the muscle of the stomach; and (c) the cyst wall is lined by the epithelium of the alimentary tract. The lesions found in our case fulfilled these criteria for GDCs. Most cases of GDC are diagnosed in the first year of life and with vomiting and a palpable abdominal mass. It is unusual for GDCs to remain asymptomatic until adulthood, and the patient in our case is one of the oldest GDC patients to be diagnosed in adulthood to date.

GDCs are classified as either cystic or tubular depending on their communication and contiguity with the stomach. More than $80 \%$ of gastric duplications are cystic and do not communicate with the gastric lumen. The remaining $20 \%$ comprise tubular GDCs, which are contiguous with the stomach and communicate to a certain extent with its lumen [13]. Duplication cysts of the ileum are usually located on the mesenteric border, whereas the usual location for GDCs is along the greater curvature, followed by the posterior wall of the stomach. This is because these duplications are usually distributed dorsal to the primitive gut during development. GDCs are located along the lesser curvature only in 5.5\% of reported cases, and the mechanism by which they develop is not well understood [19]. In our case, the tubular-shaped GDC was located along the lesser curvature of the stomach and communicated with the gastric lumen. Within the root of the GDC, the lumen was surrounded by the gastric mucosa and smooth muscle.

Adenocarcinoma arising from a GDC is extremely rare with only 16 cases reported in the literature (Table 1) [2-16]. As shown in Table 1, carcinomas arising from GDCs generally occur in middle-aged adults and are 
diagnosed as large-sized masses. The probability of malignant transformation of GDCs increases with age. Persistent exposure to carcinogenic substances such as salty pickles and Helicobacter pylori infection is considered to be the cause of malignant transformation. However, almost all adenocarcinomas arising from GDCs are cystic, without communication with the gastric lumen. This case is the first report of adenocarcinoma originating from a GDC and communicating with the lesser curvature of the stomach. The patient was diagnosed with a Helicobacter pylori infection by a stool antigen test and subsequently underwent eradication therapy. Helicobacter pylori-induced gastritis may have led to gastric cancer in this case. Up to $10 \%$ of GDCs may contain ectopic pancreatic tissue, which may lead to pancreatitis that mimics a pancreatic pseudocyst. However, malignant transformation of heterotopic pancreatic tissue is extremely rare. Persistent irritation together with an increase in the intracystic pressure and oxygen deficiency may cause chronic inflammation, repeated apoptosis, and regeneration of the epithelium, which may ultimately lead to malignant transformation within the GDC [11]. In this situation, complications such as obstruction, necrosis, and infection were possible, but the patient did not have these complications until adulthood.

\begin{tabular}{|c|c|c|c|c|c|c|c|c|c|c|}
\hline Reference & Age & Sex & Symptoms & Location & Communication & CA19-9(U/ml) & Histology & Size & Surgery & Outcome \\
\hline Mayo et al. [2] & 64 & $\mathrm{~F}$ & Weakness, anorexia & GC/Antrum & No & NA & tub1 & $6 \mathrm{~cm}$ & Distal gastrectomy & $12 \mathrm{~m} /$ Alive \\
\hline Coit et al. [3] & 72 & $F$ & Pain, weight loss & GC & No & NA & muc/pap & $3.2 \mathrm{~cm}$ & Distal gastrectomy & 72m/Alive \\
\hline Kuraoka et al. [4] & 40 & $M$ & Fever, backpain & GC & No & NA & ADC & $7 \mathrm{~cm}$ & Proximal gastrectomy & $7 \mathrm{~m} /$ Alive with recurrence \\
\hline Horne et al. [5] & 40 & $\mathrm{M}$ & Pain & GC & No & $\mathrm{NA}$ & NEC & $12 \mathrm{~cm}$ & Total gastrectomy+DPS $+\mathrm{H}$ & $14 \mathrm{~m} / \mathrm{Alive}$ with recurrence \\
\hline Barussaud et al. [6] & 67 & $\mathrm{~F}$ & Mass, weight loss & Antrum & $\mathrm{NA}$ & 280 & ADC/SCC & $18 \mathrm{~cm}$ & Distal gastrectomy & $6 \mathrm{~m} /$ Lead \\
\hline Fukumoto et al. [7] & 50 & $\mathrm{M}$ & Vomiting & Duodenum & No & 297 & tub1/por & $3 \mathrm{~cm}$ & Local Excision, PD & Dead \\
\hline Zheng et al. [8] & 25 & $\mathrm{M}$ & Asymptomatic & GC & No & NA & ADC & $8 \mathrm{~cm}$ & Total gastrectomy & 13m/Alive \\
\hline Kang et al. [9] & 56 & $\mathrm{M}$ & Asymptomatic & GC/Corpus & No & $\mathrm{NA}$ & $\mathrm{ADC}$ & $5.5 \mathrm{~cm}$ & Local Excision & NA \\
\hline Liu et al. [10] & 28 & M & Asymptomatic & GC/Corpus & No & 2145 & $A D C$ & $13 \mathrm{~cm}$ & Local Excision & $7 \mathrm{~m} /$ Alive with metastasis \\
\hline \multirow[t]{2}{*}{ Zhu et al. [11] } & 62 & $\mathrm{M}$ & Pain & GC & No & NA & NEC & $4 \mathrm{~cm}$ & Total gastrectomy & NA \\
\hline & 72 & $\mathrm{M}$ & Regurgitation & Posterior & No & NA & NEC & $2 \mathrm{~cm}$ & Distal gastrectomy & NA \\
\hline Yamasaki et al. [12] & 47 & $\mathrm{~F}$ & Asymptomatic & GC & No & 167.1 & tub2 & $10 \mathrm{~cm}$ & Local Excision & $9 \mathrm{~m} /$ Dead \\
\hline Abdulla et al. [13] & 51 & $\mathrm{M}$ & Melena & GC & Yes & NA & tub2 & $5 \mathrm{~cm}$ & Total gastrectomy & NA \\
\hline Han et al. [14] & 41 & $\mathrm{~F}$ & NA & Posterior & No & NA & tub2/SCC & $6 \mathrm{~cm}$ & Total gastrectomy+DP & NA \\
\hline Sethi et al. [15] & 63 & $\mathrm{M}$ & Vomiting & Antrum & No & WNL & pap & $10 \mathrm{~cm}$ & Distal gastrectomy $+\mathrm{H}$ & NA \\
\hline Chang et al. [16] & 57 & $\mathrm{~F}$ & Abdominal discomfort & Posterior & No & WNL & tub2 & 8.2 & Total gastrectomy & 6m/Alive \\
\hline Present case & 72 & $\mathrm{~F}$ & Asymptomatic & LC & Yes & 512.38 & tub2 & $11 \mathrm{~cm}$ & Distal gastrectomy & 4y/Alive \\
\hline
\end{tabular}

TABLE 1: Clinicopathological data of patients diagnosed with carcinoma arising from a gastric duplication cyst.

NA: not available; GC: greater curvature; LC: lesser curvature; WNL: within normal limits; tub1: well-differentiated adenocarcinoma; tub2: moderately differentiated adenocarcinoma; muc: mucinous carcinoma; pap: papillary adenocarcinoma; ADC: adenocarcinoma; NEC: neuroendocrine carcinoma; SCC: squamous cell carcinoma; DPS: distal pancreatosplenectomy; H: hepatectomy; PD: pancreaticoduodenectomy; DP: distal pancreatectomy; mo: month; y: year

Gastritis cystica profunda (GCP) is one of the most important differential diagnoses. GCP usually develops in the stomach after gastrectomy and is considered to be a precancerous lesion associated with Epstein-Barr virus infection. Adenocarcinoma rarely arises from GCP in the unoperated stomach. The difference between adenocarcinoma arising from a GDC and that arising from GCP is the presence of a smooth muscle coat surrounding the lesion in case of the former [9]. A gastrointestinal stromal tumor (GIST) is also an important differential diagnosis because the stomach is a common location for GISTs. GISTs usually present as solid tumors; however, sometimes large areas of internal hemorrhage and cystic degeneration may occur [14]. CT is useful in detecting the lesion and determining its relationship with other organs. EUS and EUS-guided fine-needle aspiration can provide a more definitive diagnosis. PET/CT was also useful in distinguishing between benign and malignant lesions in this case. Estimating serum CA 19-9 levels may be useful in predicting malignancy 
in some cystic lesions such as ovarian cysts or intraductal papillary mucinous neoplasms of the pancreas. In gastric cancer, elevated serum CA 19-9 levels are predictive of lymph node metastasis [20]. Among the reviewed cases, including the present case, the serum CA 19-9 levels were elevated in five cases. Barring our case, the four remaining cases showed early recurrence and a poor prognosis. In gastric cancer originating from GDCs, the depth of invasion is not always associated with the size of the GDCs. In almost all reported cases, stomach resection surgery was performed. Moreover, the long-term prognosis after local excision of the GDC has not been confirmed. GDCs in adults, especially lesions with elevated serum CA 19-9 levels, should be managed surgically via gastric resection and regional lymphadenectomy [7].

This is the only case in which the long-term prognosis over a four-year-period has been reported after surgery for gastric cancer with lymph node metastasis arising from a GDC. In most cases, tumors without communication with the gastric lumen grow asymptomatically. By the time symptoms appear, such tumors have already invaded the surrounding organs or metastasized. Although the diagnosis of malignancy originating from a GDC is difficult, once a GDC is detected, even if it is asymptomatic, it should be removed, considering its potential for malignancy. We should be careful not to rupture the cyst wall as this will result in peritoneal carcinomatosis.

\section{Conclusions}

In conclusion, GDCs are rare and usually present as benign lesions but may predispose to malignancy and other complications in adults. GDCs should be considered in the differentiation of cystic masses of the gastrointestinal tract. Once detected, prompt surgical intervention is important for optimal outcomes.

\section{Additional Information \\ Disclosures}

Human subjects: Consent was obtained by all participants in this study. Conflicts of interest: In compliance with the ICMJE uniform disclosure form, all authors declare the following: Payment/services info: All authors have declared that no financial support was received from any organization for the submitted work. Financial relationships: All authors have declared that they have no financial relationships at present or within the previous three years with any organizations that might have an interest in the submitted work. Other relationships: All authors have declared that there are no other relationships or activities that could appear to have influenced the submitted work.

\section{Acknowledgements}

We would like to thank Editage (www.editage.com) for English language editing.

\section{References}

1. GMH Veeneklaas: Pathogenesis of intrathoracic gastrogenic cysts . Am J Dis Child. 1952, 83:500-7. 10.1001/archpedi.1952.02040080096010

2. Mayo HW Jr, McKee EE, Anderson RM: Carcinoma arising in reduplication of the stomach (gastrogenous cyst). Ann Surg. 1955, 141:550-5. 10.1097/00000658-195504000-00019

3. Coit DG, Mies C: Adenocarcinoma arising within a gastric duplication cyst . J Surg Oncol. 1992, 50:274-7. 10.1002/jso.2930500417

4. Kuraoka K, Nakayama H, Kagawa T, Ichikawa T, Yasui W: Adenocarcinoma arising from a gastric duplication cyst with invasion to the stomach: a case report with literature review. J Clin Pathol. 2004, 57:428-31. 10.1136/jcp.2003.013946

5. Horne G, Ming-Lum C, Kirkpatrick AW, Parker RL: High-grade neuroendocrine carcinoma arising in a gastric duplication cyst: a case report with literature review. Int J Surg Pathol. 2007, 15:187-91. $10.1177 / 1066896906295777$

6. Barussaud ML, Meurette G, Cassagnau E, Dupas B, Le Borgnea J: Mixed adenocarcinoma and squamous cell carcinoma arising in a gastric duplication cyst. Gastroenterol Clin Biol. 2008, 32:188-91. 10.1016/j.gcb.2008.01.014

7. Fukumoto K, Suzuki S, Sakaguchi T, et al.: Adenocarcinoma arising from gastric duplication: a case report with literature review. Clin J Gastroenterol. 2008, 1:148-52. 10.1007/s12328-008-0024-1

8. Zheng J, Jing H: Adenocarcinoma arising from a gastric duplication cyst . Surg Oncol. 2012, 21:e97-101. 10.1016/j.suronc.2012.03.002

9. Kang HJ, Jang SJ, Park YS: Adenocarcinoma arising in gastric duplication cyst . Korean J Pathol. 2014, 48:159-61. 10.4132/KoreanJPathol.2014.48.2.159

10. Liu K, Lin X, Wu J, et al.: Peritoneal metastatic adenocarcinoma possibly due to a gastric duplication cyst: a case report and literature review. BMC Gastroenterol. 2014, 14:48. 10.1186/1471-230X-14-48

11. Zhu Y, Lihong LV, Pan W, Ren P, Han T, Xu X: Gastric duplication complicated by malignant transformation in adults: report of three cases. J Gastrointest Dig Syst. 2015, 5:374. 10.4172/2161-069X.1000374

12. Yamasaki A, Onishi H, Yamamoto H, et al.: Asymptomatic adenocarcinoma arising from a gastric duplication cyst: a case report. Int J Surg Case Rep. 2016, 25:16-20. 10.1016/j.ijscr.2016.05.055

13. Abdulla MAM, Al Saeed M, Ameer Alshaikh S, Nabar UJ: Adenocarcinoma arising from a gastric duplication cyst: a case report and literature review. Int Med Case Rep J. 2017, 10:367-72. 10.2147/IMCRJ.S138616

14. Han SY, Kim GH: Collision tumor arising from a gastric duplication cyst . Gastrointest Endosc. 2017, 86:7389. 10.1016/j.gie.2017.03.1543 


\section{Cureus}

15. Sethi S, Godhi S, Puri SK: Papillary adenocarcinoma in a gastric duplication cyst . Indian J Surg Oncol. 2018, 9:79-82. 10.1007/s13193-017-0714-6

16. Chan BH, Hyrcza M, Ramsay J, Tse F: Adenocarcinoma arising from a gastric duplication cyst . ACG Case Rep J. 2018, 5:e42. 10.14309/crj.2018.42

17. Ladd WE: Duplications of the alimentary tract. Sth Med J. 1937, 30:363-71. 10.1097/00007611-19370400000002

18. Lewis PL, Holder T, Feldman M: Duplication of the stomach. Report of a case and review of the English literature. Arch Surg. 1961, 82:634-40. 10.1001/archsurg.1961.01300100148017

19. Tjendra Y, Lyapichev K, Henderson J, Rojas CP: Foregut duplication cyst of the stomach: a case report duplication cyst in an adult and review of the literature. Case Rep Pathol. 2016, 2016:7318256. 10.1155/2016/7318256

20. Yu J, Zhang S, Zhao B: Differences and correlation of serum CEA, CA19-9 and CA72-4 in gastric cancer . Mol Clin Oncol. 2016, 4:441-9. 10.3892/mco.2015.712 